\title{
Learning Effect of Humphrey Matrix Frequency Doubling Technology Perimetry in Patients With Ocular Hypertension
}

\author{
Marco Centofanti, MD, PhD, ${ }^{\dagger}$ Paolo Fogagnolo, MD, $\dagger$ Francesco Oddone, MD, $\dagger$ \\ Nicola Orzalesi, MD, + Michele Vetrugno, MD, $\S$ Gianluca Manni, MD, ${ }^{*} \dagger$ and Luca Rossetti, MD $\ddagger$
}

\begin{abstract}
Aim: To evaluate the learning effect of Frequency Doubling Technology (FDT) perimetry using the Humphrey Matrix-FDT perimetry (Matrix) 24-2 full-threshold program on patients with ocular hypertension experienced with standard automated perimetry.

Methods: Twenty-four patients with ocular hypertension underwent 5 full-threshold Matrix tests at intervals of $5 \pm 2$ days. Learning effect was defined as an improvement at results for duration, perimetric indices, foveal sensitivity, Glaucoma Hemifield Test, and the number of points with a $P<5 \%$ and $<1 \%$ in the total and pattern deviation maps. Eccentricity, hemifield, and quadrant sensitivities were also addressed as sources of differences in learning effect. Test-retest variability was also calculated for each repetition as the mean of the pointto-point interindividual standard deviations.
\end{abstract}

Results: A learning effect was demonstrated for mean defect $(P=0.031$, analysis of variance) and foveal sensitivity $(P=0.009)$ and it only affected the first test for both parameters. All the other parameters did not show any significant learning effect. The effect was independent from eccentricity and quadrant or hemifield sensitivities.

Conclusions: The results of this study demonstrate that the learning effect for Matrix-FDT is mild and it may affect only the first test. Caution is needed in the analysis of the first MatrixFDT examination and retest may be advisable in the presence of low mean defect.

Key Words: frequency doubling technology, Matrix, perimetry, learning effect, ocular hypertension, glaucoma, test-retest variability

( J Glaucoma 2008;17:436-441)

Received for publication July 12, 2007; accepted October 20, 2007.

From the *Department of Ophthalmology, "Tor Vergata" University of Rome; $\dagger$ G.B. Bietti Foundation for the Study and Research in Ophthalmology, IRCCS, Rome; †Eye Clinic, San Paolo Hospital,

University of Milan, Milan; and §Department of Ophthalmology, University of Bari, Bari, Italy.

Sources of support that require acknowledgment: None.

Reprints: Marco Centofanti, MD, PhD, Department of Ophthalmology,

"Tor Vergata" University of Rome, Via di Trasone 6, 00199, Roma,

Italy (e-mail: mcentofanti@katamail.com).

Copyright (c) 2008 by Lippincott Williams \& Wilkins
$T^{\mathrm{h}}$ he improvement in perimetric results occurring over repetitions in inexperienced subjects is a well-known phenomenon called "learning effect." Learning is an important factor limiting the validity of the first tests obtained with standard automated perimetry (SAP) ${ }^{1}$ and nonconventional perimetries such as short-wavelength automated perimetry (SWAP)., ${ }^{2,3}$ On the opposite, the learning effect for both screening ${ }^{4-6}$ and full-threshold ${ }^{7-11}$ programs of the first generation of Humphrey Frequency Doubling Technology (FDT) perimetry has been shown to be mild and it could be minimized by previous experience with other automated perimetries.

A new version of FDT perimetry, called Matrix, has been recently introduced in the clinical practice. The diagnostic performances of Matrix have been up-to-now evaluated only in a small number of clinical studies: a good correlation between results at Matrix and the Swedish Interactive Threshold Algorithm standard test (SITA-SAP) has been shown ${ }^{12}$ and its sensitivity is probably at least similar to that of the first-generation FDT. ${ }^{13}$ Also, a recent cross-sectional study showed that Matrix achieved the best diagnostic power for early glaucoma when compared with single morphologic tests (scanning laser polarimetry, Optical Coherence Tomography, and retinal nerve fiber layer photography). ${ }^{14}$ Nevertheless, the limits of this perimetry have only been partially explored: in particular, its learning effect has only been evaluated by Contestabile and aggregates. ${ }^{15}$ In this nonrandomized study conducted over 37 healthy subjects, inexperienced to perimeter, undergoing 2 repetitions in both eyes, a significant learning effect was found only for the first repetition. Still, to exclude the possibility of a further improvement of performances, the learning effect should have been explored over a larger number of tests and possibly on a group of patients representing the recipient of the technique (ie, patients at risk for glaucoma or with initial disease).

We, therefore, addressed the Matrix learning effect over a battery of 5 tests on patients at risk for developing glaucoma and already experienced with SAP.

\section{MATERIALS AND METHODS}

The study involved 24 subjects attending the Glaucoma Services of 3 Italian University Eye Clinics: 
Rome Tor Vergata, the University of Bari, and San Paolo Hospital (Milan). The study protocol was peer reviewed by the Institutional Review Board and it was approved by the Centres' Ethics Committees; it adhered to the principles of the Declaration of Helsinki. After careful explanation of the procedures of the study and of the nature of the FDT stimulus, enrolled patients gave their written informed consent.

Inclusion criteria were the following: (1) ocular hypertension, both treated or untreated, defined as the presence of an intraocular pressure (IOP) $>21 \mathrm{~mm} \mathrm{Hg}$ without medication ( 2 consecutive Goldmann tonometer measurements separated by an interval of at least $2 \mathrm{~h}$ but no more than $12 \mathrm{wk}$ ). (2) No changes in IOP treatment during the course of the study. (3) Absence of optic nerve head defects (evaluated by indirect ophthalmoscopy using a +90 -D condensing lens). (4) Normal retinal nerve fiber layer (absence of slit or wedge defects at scanning laser ophthalmoscopy ${ }^{16}$ or normality in all sectors at scanning laser polarimetry). ${ }^{17}$ (5) SAP experience (at least 4 previous tests). (6) Normal 30-2 Swedish Interactive Threshold Algorithm standard test-SAP, defined on the basis of the criteria proposed by Hodapp et al. ${ }^{18}$ Normality was defined on the basis of at least 2 consecutive and reliable SAP tests. (7) No previous FDT experience (both Matrix and first-generation FDT). (8) Corrected visual acuity of 20/40 or better. (9) Refraction between -5 and $+5 \mathrm{D}$ and astigmatism less than \pm 3 D. (10) Pupils more than $3 \mathrm{~mm}$ in diameter. (11) Clear media. (12) No previous eye surgery. (13) Absence of any retinal abnormalities. Table 1 summarizes the patients' main characteristics.

TABLE 1. Demographic and SAP Indices of Study Participants

\begin{tabular}{lc}
\hline No. subjects & 24 \\
Age, y (SD) & $56.3(8.2)$ \\
Range & $31 ; 69$ \\
Sex & $13 \mathrm{~F}, 11 \mathrm{M}$ \\
CCT, $\mu \mathrm{m}$ (SD) & $556(13)$ \\
Range & $535 ; 572$ \\
Refraction, D (SD) & $+0.67(0.93)$ \\
Range & $-1.50 ;+2.75$ \\
Baseline IOP, mm Hg (SD) & $23.3(2.8)$ \\
Range & $21 ; 29$ \\
Best corrected visual acuity (SD) & $20 / 20(20 / 120)$ \\
Range & $20 / 20 ; 20 / 16$ \\
Treatment & \\
$\quad$ None & 1 \\
$\quad \beta$-blockers & 16 \\
Prostaglandins & 6 \\
$\quad \alpha$-agonists & 3 \\
Actual IOP, mm Hg (SD) & $16.6(1.8)$ \\
Range & $13 ; 20$ \\
SAP MD, dB (SD) & $-0.65(1.18)$ \\
Range & $-2.69 ;+0.87$ \\
SAP PSD, dB (SD) & $1.78(0.69)$ \\
Range & $0.77 ; 2.92$ \\
\hline
\end{tabular}

CCT indicates central corneal thickness; IOP, intraocular pressure; MD, mean defect; PSD, pattern standard deviation; SAP, standard automated perimetry; SD, standard deviation.
A review of the medical charts confirmed that at least the last 3 SAP repetitions did not encounter any significant change (ie, learning effect): no expansion or reduction of the clusters of abnormal locations, no changes in the mean deviation (MD) and pattern standard deviation (PSD) probability value and in the Glaucoma Hemifield Test (GHT) were found.

For the purpose of the study, the patients underwent a complete ophthalmic examination (anterior segment examination, gonioscopy, Goldmann applanation tonometry, dilated posterior segment examination with a $+90-\mathrm{D}$ condensing lens and indirect ophthalmoscopy, and pachimetry). Thereafter, 1 eye of each patient was randomized to receive an SAP test to confirm diagnosis, an FDT demo test, and 5 Matrix tests at intervals of $5 \pm 2$ days. All of the visual field tests were performed by a single operator in each study centre.

Matrix was performed using the 24-2 full-threshold program of the commercially available Humphrey FDT (Carl Zeiss Meditec, Dublin, CA). Stimulus characteristics of the Matrix FDT Program 24-2 test are similar to those previously described for the first-generation FDT device, ${ }^{19}$ being 5 -degree square with spatial frequency of 0.5 cycles/degree and temporal frequency of $18 \mathrm{~Hz}$. A Zippy Estimation by Sequential Testing thresholding strategy is used with a flat previous probability density function and fixed termination criterion. ${ }^{20}$

To be included in the analysis, all of the Matrix tests had to obtain reliability indices within normal limits (fixation losses $<25 \%$, false positive $<33 \%$, and false negative responses $<33 \%$ ); if this was not the case, the test was repeated. Two patients needed 1 repetition owing to an abnormal number of fixation losses. All subjects completed all visits.

A learning effect was defined in the presence of improvement in duration, MD, PSD, foveal sensitivity, GHT, and number of locations with $<5 \%$ and $P<1 \%$ in both total and pattern deviation maps. Mean central, paracentral, and peripheral sensitivities (respectively 5, 28, and 36 locations; Fig. 1), and mean quadrant and hemifield sensitivities (defined as the mean sensitivity of the locations in each area) were also calculated and compared to detect possible differences in the learning effect. Test-retest variability (TRV) was also calculated for each repetition as the mean of the point-to-point interindividual standard deviations (after that the pointto-point sensitivities for left eyes had been converted as if they were right eyes).

Improvement over repetitions was evaluated by means of linear regression and analysis of variance (ANOVA) for repeated measures; the Bonferroni correction was considered. For parameters with statistically significant ANOVA, matched-paired $t$ test $(\alpha=0.05)$ was used to detect interexamination differences. For GHT (a qualitative parameter), $\chi^{2}$ test was used. We also inspected the correlations between the learning effect (arbitrarily defined, for each patient, as the difference of the first minus that of the fifth test for both MD and PSD) and age, sex, eye, refraction, baseline IOP, current IOP, 


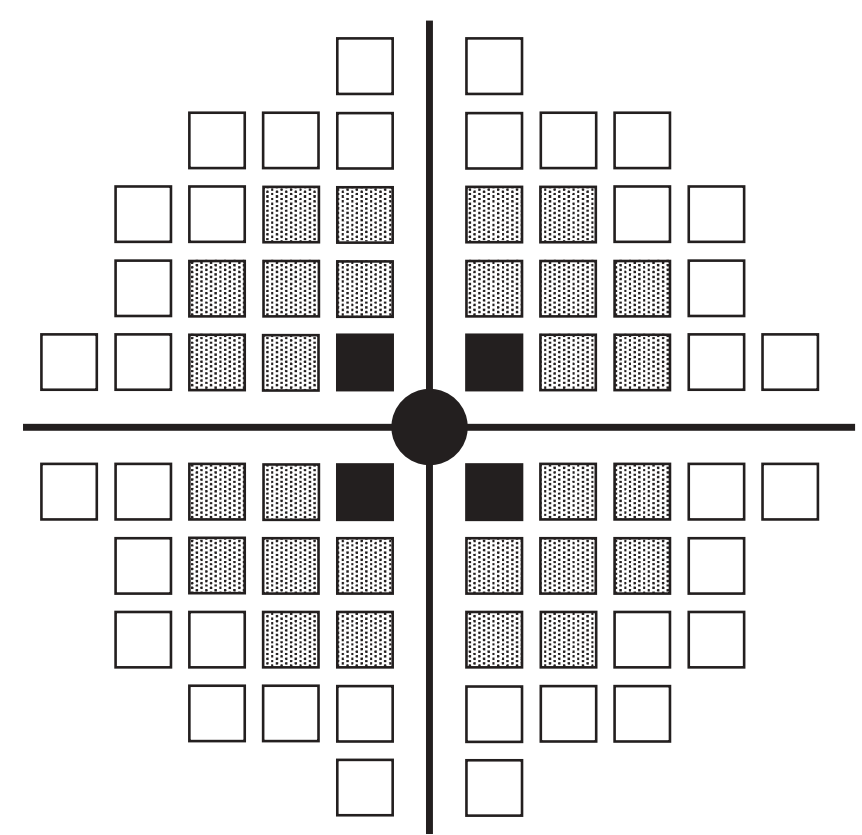

FIGURE 1. Matrix locations were divided in central (black squares), paracentral (gray), and peripheral (white) ones to study the role of eccentricity on learning effect.

central corneal thickness, and MD at the first and fifth tests using the Bravais-Pearson correlation coefficient $(\rho)$. The correlations were negligible if $\rho \leq 0.2$, weak if $0.2<\rho \leq 0.5$, good if $0.5<\rho \leq 0.8$, and strong if $\rho>0.8$.

\section{RESULTS}

The results of the study are summarized in Table 2 (average results for each repetition) and Table 3 (percentage of improving patients between the first and fifth test and between the second and the fifth test).

Mean defect (Fig. 2) progressively decreased from $-0.74 \pm 3.10 \mathrm{~dB}$ at the first examination to $+0.18 \pm 3.10 \mathrm{~dB}$ at the fifth examination $(P=0.031$, ANOVA), with a mean improvement of about $0.2 \mathrm{~dB}$ per test $\left(R^{2}=0.94, P=0.001\right)$. Yet, only the first test obtained statistically different results from the others (first vs. fourth: $P=0.003$ and first vs. fifth: $P=0.005$; $t$ test). Overall, $71 \%$ of subjects obtained better MD results when comparing the fifth and the first examination.

Also foveal sensitivity (Fig. 3) significantly increased from $29.1 \pm 3.0 \mathrm{~dB}$ at the first examination to $31.0 \pm 4.1 \mathrm{~dB}$ at the fifth examination $(P=0.009$, ANOVA), with a mean improvement of about $0.5 \mathrm{~dB}$ per test $\left(R^{2}=0.70, P=0.004\right)$. Once again, the only differences were found between the first and the fourth tests $(P=0.010, t$ test $)$, and the first and the fifth tests $(P=0.023)$. Overall, foveal sensitivity improved in $92 \%$ of patients between the first and the fifth examinations.

A similar behavior of Matrix was obtained between repetitions when evaluating TRV (Fig. $4, P<0.0001$, ANOVA), with the first test being significantly different from the fourth $(P=0.02)$ and the fifth $(P=0.005)$.

No significant variations were found in duration (which was $6.15 \mathrm{~min}, \quad P=0.22, \quad$ ANOVA), PSD $(P=0.78, \quad$ ANOVA $)$, number of abnormal GHT

TABLE 2. Mean Data (SD) at Each Matrix Repetition

\begin{tabular}{|c|c|c|c|c|c|}
\hline & \multicolumn{5}{|c|}{ Matrix Test } \\
\hline & First & Second & Third & Fourth & Fifth \\
\hline Duration (min) & $6.17(0.11)$ & $6.16(0.14)$ & $6.18(0.16)$ & $6.13(0.13)$ & $6.16(0.14)$ \\
\hline $\mathrm{MD}(\mathrm{dB})$ & $-0.74(3.10)^{* \dagger}$ & $-0.44(3.63)$ & $-0.44(3.78)$ & $-0.16(3.13)$ & $0.18(3.10)$ \\
\hline PSD (dB) & $2.67(0.35) \div \S$ & $2.72(0.71)$ & $2.82(0.72)$ & $2.86(0.84)$ & $2.77(0.63)$ \\
\hline Abnormal GHT & $6 / 24$ & $3 / 24$ & $9 / 24$ & $5 / 24$ & $4 / 24$ \\
\hline Borderline GHT & $3 / 24$ & $3 / 24$ & $5 / 24$ & $2 / 24$ & $6 / 24$ \\
\hline No. points with $P<5 \%$ (total deviation map) & $4.1(6.3)$ & $4.3(7.0)$ & $4.3(6.2)$ & $2.8(6.3)$ & $2.6(4.0)$ \\
\hline No. points with $P<5 \%$ (pattern deviation map) & $0.5(0.9)$ & $1,0(1.7)$ & $1.1(2.0)$ & $0.8(1.5)$ & $0.7(1.8)$ \\
\hline No. points with $P<1 \%$ (total deviation map) & $2.2(2.2)$ & $2.3(2.1)$ & $2.9(2.5)$ & $2.7(3.4)$ & $3.0(3.1)$ \\
\hline No. points with $P<1 \%$ (pattern deviation map) & $0.7(1.0)$ & $1.2(2.2)$ & $1.1(1.9)$ & $1.4(2.4)$ & $1.1(2.0)$ \\
\hline Foveal sensitivity $(\mathrm{dB})$ & $29.1(3.0)$ & $30.6(4.0)$ & $30.7(5.1)$ & $31.0(3.3)$ & $31.0(4.1)$ \\
\hline Mean sensitivity $(\mathrm{dB})$ & $25.55(4.60)$ & $25.89(4.92)$ & $25.92(5.25)$ & $26.21(4.69)$ & $26.63(4.61)$ \\
\hline Central sensitivity $(\mathrm{dB})$ & $29.2(3.0)$ & $29.9(3.7)$ & $30.0(4.5)$ & $30.4(2.9)$ & $30.5(3.5)$ \\
\hline Paracentral sensitivity (dB) & $26.5(3.5)$ & $26.4(4.0)$ & $26.6(4.0)$ & $26.8(3.5)$ & $27.2(3.6)$ \\
\hline Peripheral sensitivity $(\mathrm{dB})$ & $24.4(3.2)$ & $24.9(3.7)$ & $24.8(4.0)$ & $25.2(3.4)$ & $25.7(3.1)$ \\
\hline Superonasal quadrant sensitivity (dB) & $24.8(3.3)$ & $25.2(3.7)$ & $25.3(4.1)$ & $25.4(3.6)$ & $26.1(3.4)$ \\
\hline Superotemporal quadrant sensitivity (dB) & $25.9(3.0)$ & $26.0(3.9)$ & $25.9(4.1)$ & $26.3(3.2)$ & $26.6(3.0)$ \\
\hline Inferonasal quadrant sensitivity $(\mathrm{dB})$ & $25.7(3.7)$ & $26.0(4.0)$ & $26.1(4.5)$ & $26.4(3.7)$ & $26.7(3.7)$ \\
\hline Inferotemporal quadrant sensitivity (dB) & $25.6(3.8)$ & $26.0(3.8)$ & $26.1(4.0)$ & $26.4(3.7)$ & $26.9(3.5)$ \\
\hline Total TRV $(\mathrm{dB})$ & $3.3(0.4) \| \oplus$ & $3.1(0.6)$ & $3.1(0.5)$ & $3.0(0.5)$ & $3.0(0.4)$ \\
\hline Range of TRV (dB) & $2.7 ; 3.8$ & $2.2 ; 4.2$ & $2.3 ; 3.8$ & $2.4 ; 4.3$ & $2.4 ; 3.9$ \\
\hline
\end{tabular}

*First vs. fourth: $P=0.003$

$\dagger$ First vs. fifth: $P=0.005$.

First vs. fourth: $P=0.010$.

$\S$ First vs. fifth: $P=0.023$.

$\|$ First vs. fourth: $P=0.02$.

TFirst vs. fifth: $P=0.005$.

MD indicates mean defect; PSD, pattern standard deviation; brackets represent standard deviations. 
TABLE 3. Proportion, Percentage, and 95\% Confidence Intervals (Cls) of Improving Patients

\begin{tabular}{|c|c|c|c|c|}
\hline \multirow[b]{2}{*}{ Parameter } & \multicolumn{2}{|c|}{ First vs. Fifth Matrix } & \multicolumn{2}{|c|}{ Second vs. Fifth Matrix } \\
\hline & Percentage $(\%)$ & $95 \%$ CIs & Percentage $(\%)$ & 95\% CIs \\
\hline Duration & 50 & $30 \%-70 \%$ & 37 & $18 \%-57 \%$ \\
\hline MD & 71 & $53 \%-82 \%$ & 62 & $43 \%-82 \%$ \\
\hline Abnormal GHT & 75 & $58 \%-92 \%$ & 54 & $34 \%-74 \%$ \\
\hline Foveal sensitivity & 92 & $81 \%-100 \%$ & 42 & $22 \%-61 \%$ \\
\hline No. points with $P<5 \%$ (total deviation map) & 79 & $63 \%-95 \%$ & 33 & $14 \%-52 \%$ \\
\hline No. points with $P<1 \%$ (pattern deviation map) & 75 & $58 \%-92 \%$ & 33 & $14 \%-52 \%$ \\
\hline TRV & 58 & $38 \%-78 \%$ & 58 & $38 \%-78 \%$ \\
\hline
\end{tabular}

Significant improvements are in bold characters.

$\left(P=0.50, \chi^{2}\right.$ test $)$, and number of abnormal locations for total and pattern deviation maps, both at $P<5 \%$ and $<1 \%$ (respectively $P=0.30,0.53,0.52$, and 0.57 , ANOVA).

Homogeneity of learning effect throughout all locations was demonstrated, as it was independent from eccentricity (comparison between central, paracentral, and peripheral locations was not significant, $P=0.18$, $0.86,0.25)$, and no differences were found between hemifield and quadrant sensitivities $(P=0.19$ and $=0.23$; Table 2).

Table 3 summarizes the percentage of improving subjects between repetitions. Once again, the first test was prone to learning artefacts as significant improvements were obtained for many indices compared with control (fifth test); on the opposite, no significant changes in results were found comparing the second and the fifth tests.

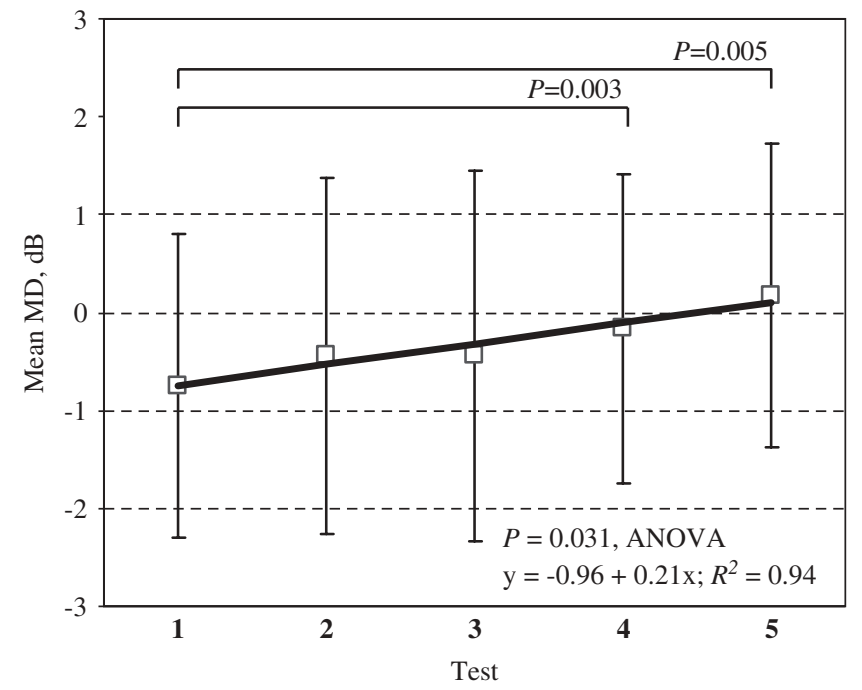

FIGURE 2. Learning effect for mean defect. Bars represent standard deviations; brackets indicate the results of the post hoc $t$ tests.
Correlations between learning effect and demographic (age, sex, baseline IOP, best corrected visual acuity, refraction) and perimetric variables (both MD and PSD) were weak or negligible and statistically not significant except for a good correlation between improvement in results for MD and actual IOP $(\rho=0.51$, $P=0.02)$.

\section{DISCUSSION}

In this study we evaluated the learning effect for the Matrix perimetry over 5 test repetitions in patients already experienced with SAP; our results confirm previous findings suggesting that learning artefacts for FDT are mild, regardless of the version of the device, of the adopted strategy (screening ${ }^{4-6}$ and full-threshold ${ }^{7-11}$ procedures), and of the features of the study population (normal subjects vs. glaucomatous patients; presence or absence of previous perimetric experience).

Our dataset was analyzed using different statistical techniques (ANOVA, regression, $t$ test for comparison of mean test results, and percentage of the individual

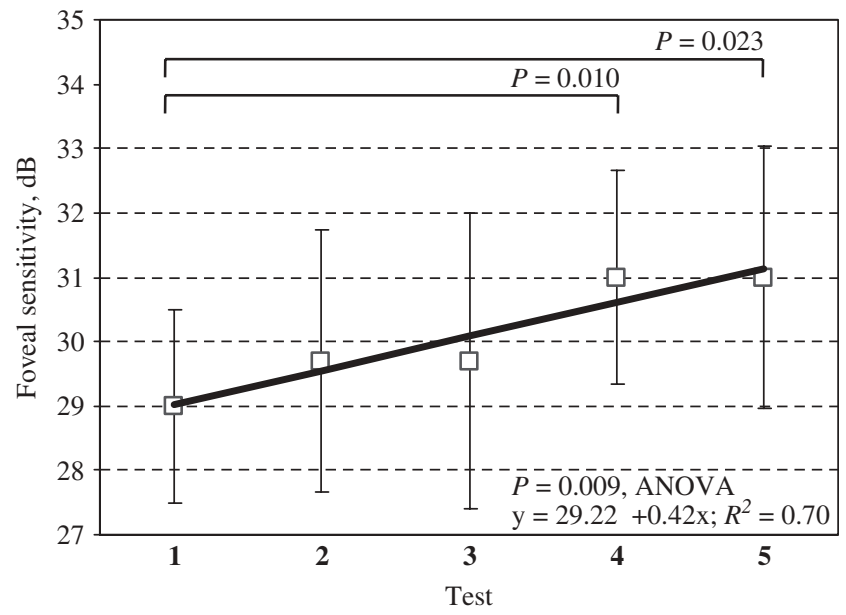

FIGURE 3. Learning effect for foveal sensitivity. Bars represent standard deviations; brackets indicate the results of the post hoc $t$ tests. 


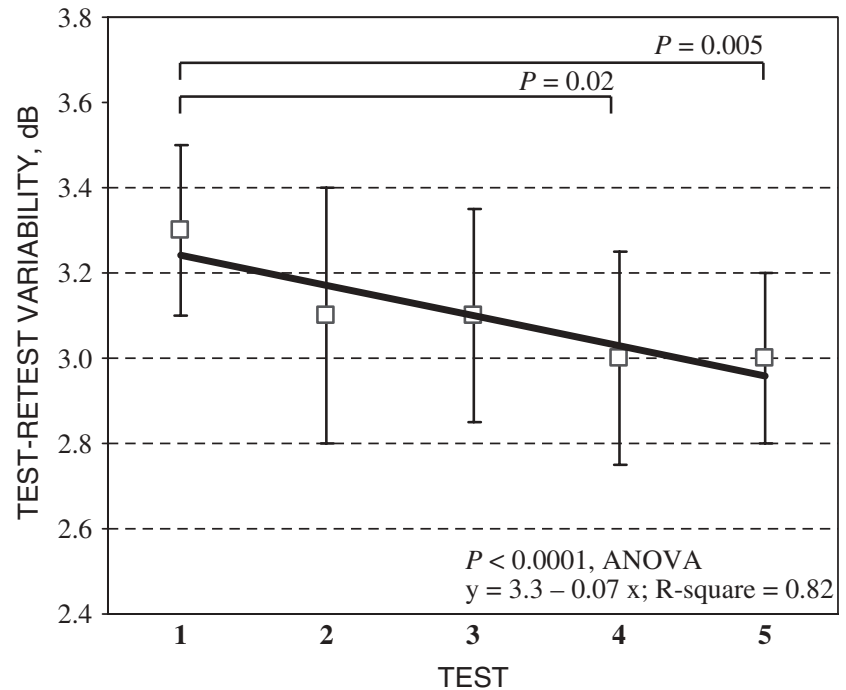

FIGURE 4. Learning effect for TRV. Bars represent standard deviations; brackets indicate the results of the post hoc $t$ tests.

improvements), and they all seemed to indicate that the learning effect could be easily ruled out by excluding the first test from analysis. In fact, considering the parameters mostly affected by learning at ANOVA (MD, foveal sensitivity, and TRV), only the first test differed from the others (Figs. 2-4); besides, only the first test obtained significant individual improvements compared with control (Table 3).

The learning effect was homogeneous throughout all perimetric locations, as it was independent from eccentricity and from quadrant and hemifield sensitivities. Correlations between the learning effect (measured as the intrapatient difference between the first and the fifth test in both MD and PSD) and many other parameters were tested. All the demographic, perimetric, and clinical data (in particular age, refraction, and best corrected visual acuity) obtained negligible correlation to the amount of learning effect. A good correlation between the improvement in MD and actual IOP $(\rho=0.51, P=0.02)$ was shown. It is the first time that such a correlation is demonstrated for any perimetric technique and this is in contrast with the findings of other authors. ${ }^{13}$ Convincing explanations for these findings are lacking and confirmations are therefore required. If the learning might be somewhat influenced by the stage of the disease, we would have expected it to significantly correlate to the baseline and not the actual IOP. At the time of the study, 23/24 patients were taking antiglaucoma medications; we could postulate that those using $\beta$-blockers and $\alpha$-adrenergics may be more prone to systemic side effects negatively influencing their performances during the first tests. Finally, a statistically significant result may also be obtained by chance.

Most of the studies on first-generation FDT enrolled normal subjects with no perimetric experience, as their aim was to address the usefulness of FDT for screening purpose. Actually, Matrix is a longer and more complex test whose primary aims are to evaluate glaucoma suspects negative to SAP ("preperimetric" glaucoma cases) or patients at risk for glaucoma and to detect the earliest progressions. Hence, we decided to include in this study a homogeneous group of ocular hypertensive eyes of SAP-experienced patients, who may ideally represent the target population for Matrix. We decided not to include patients with manifest glaucoma because we think that the presence of an additive learning effect to FDT may be a secondary problem in this group, as they undergo frequent field repetitions and they will therefore gain experience of a new perimetric technique in a relatively short time; yet, this is a critical problem in subjects with suspected glaucoma or who are at risk for developing the disease.

As our study design ruled out the presence of a SAP learning effect, the observed learning artefacts were directly related to the different nature of the stimuli used by FDT, thus confirming previous findings showing an additive learning effect for full-threshold, first-generation FDT in glaucomatous patients already experienced with SAP. ${ }^{21}$ As for first-generation FDT and other perimetric devices, ${ }^{3}$ learning effect at Matrix in patients naive to SAP could be higher than shown by our results and therefore particular caution is needed when evaluating the first Matrix tests of these subjects; owing to its design, this study cannot clarify this issue.

Very recently, the learning effect of FDT Matrix was assessed on a group of healthy subjects, inexperienced to perimetry. ${ }^{15}$ This study confirmed the presence of a learning effect at the first test, but its design was limited by the absence of eye randomization: both eyes of each patient were tested twice over 2 sessions and learning effect was esteemed as the improvement in results for left eyes. Actually, this method does not control the possible interference of phenomena such as the cortical adaptation to FDT stimulus and the fatigue effect occurring at the end of each session. Moreover, owing to the limited number of tests, this study did not show stability in perimetric performances (ie, it showed an improvement in results between the first and the second repetition, but it did not investigate the possibility of a further learning effect), as it did in our study from the second to the fifth test.

The major limit of our study is represented by the features of patients: their ages and visual acuities are not representative of the ocular hypertensive population and perhaps too stringent inclusion criteria were applied. Our findings may also be limited by the small sample size of the study and by the lack of a control group. Even if a statistically significant difference in MD, foveal threshold sensitivity, and TRV was found for only between the first test and others, MD and foveal sensitivity (Figs. 2, 3, and Table 2) seemed to progressively improve for all 5 tests. Hence, these differences could have been proved to be significant for other repeat sessions if a larger group was studied. A clinically significant learning effect could thus be evident even after the first test. 
We recently evaluated SWAP using the same design of the present study and we observed important learning artifacts for MD, duration, and the number of points with $P<5 \%$ in the pattern deviation map. ${ }^{3}$ For the majority of patients, at least 3 repetitions were required to rule out the presence of learning effect, although a subgroup of patients needed up to 5 repetitions to provide clinically useful results. The difference in results between SWAP and FDT is probably owing to the long duration of fullthreshold SWAP and to the difficulty to detect its stimulus, although weakness in SWAP database and in the algorithm to calculate threshold sensitivity may also represent likely explanations. Moreover, Matrix was also an easier examination than SWAP: only $2 / 24$ patients $(8 \%)$ required retest owing to low reliability compared with $16 / 30(53 \%)$ for SWAP $(P=0.0005$, Fisher exact test).

In conclusion, patients with ocular hypertension and experienced with SAP showed mild learning effect at Matrix, significantly affecting only the first test. In the presence of low MD values at the first Matrix, caution in analysis is needed and retest could be advisable to exclude the possibility of a false-positive result owing to learning artifacts.

\section{REFERENCES}

1. Werner EB, Adelson A, Krupin T. Effect of patient experience on the results of automated perimetry in clinically stable glaucoma patients. Ophthalmology. 1988;95:764-767.

2. Wild JM, Kim LS, Pacey IE, et al. Evidence for a learning effect in short-wavelength automated perimetry. Ophthalmology. 2006;113:206-215.

3. Rossetti L, Fogagnolo P, Miglior S, et al. Learning effect of shortwavelength automated perimetry in patients with ocular hypertension. J Glaucoma. 2006;15:399-404.

4. Brush MB, Chen PP. Learning effect among perimetric novices with screening C-20-1 frequency doubling technology perimetry. Am J Ophthalmol. 2004;137:551-552.

5. Joson PJ, Kamantigue ME, Chen PP. Learning effects among perimetric novices in frequency doubling technology perimetry. Ophthalmology. 2002;109:757-760.
6. Khong JJ, Dimitrov PN, Rait J, et al. Can the specificity of the FDT for glaucoma be improved by confirming abnormal results? J Glaucoma. 2001;10:199-202.

7. Fujimoto $\mathrm{N}$, Minowa $\mathrm{K}$, Miyauchi $\mathrm{O}$, et al. Learning effect for frequency doubling perimetry in patients with glaucoma. $\mathrm{Am}$ J Ophthalmol. 2002;133:269-270.

8. Horani A, Frenkel S, Yahalom C, et al. The learning effect in visual field testing of healthy subjects using frequency doubling technology. J Glaucoma. 2002;11:511-516.

9. Iester M, Capris P, Pandolfo A, et al. Learning effect, short-term fluctuation, and long-term fluctuation in frequency doubling technique. Am J Ophthalmol. 2000;130:160-164.

10. Matsuo H, Tomita G, Suzuki Y, et al. Learning effect and measurement variability in frequency-doubling technology perimetry in chronic open angle glaucoma. J Glaucoma. 2002;11:467-473.

11. Spry PG, Johnson CA, McKendrick AM, et al. Variability components of standard automated perimetry and frequencydoubling technology perimetry. Invest Ophthalmol Vis Sci. 2001;42: 1404-1410.

12. Burgansky-Eliash Z, Wollstein G, Patel A, et al. Glaucoma detection with matrix and standard achromatic perimetry. $\mathrm{Br}$ J Ophthalmol. 2007;91:933-938.

13. Brusini $\mathrm{P}$, Salvetat ML, Zeppieri M, et al. Visual field testing with the new Humphrey Matrix: a comparison between the FDT N-30 and Matrix N-30-F tests. Acta Ophthalmol Scand. 2006;84:351-356.

14. Hong S, Ahn H, Ha SJ, et al. Early glaucoma detection using the Humphrey Matrix perimeter, GDx VCC, Stratus OCT, and retinal nerve fiber layer photography. Ophthalmology. 2007;114:210-215.

15. Contestabile MT, Perdicchi A, Amodeo S, et al. The influence of learning effect on frequency doubling technology perimetry (Matrix). J Glaucoma. 2007;16:297-301.

16. Miglior S, Rossetti L, Brigatti L, et al. Reproducibility of retinal nerve fiber layer evaluation by dynamics scanning laser ophthalmoscopy. Am J Ophthalmol. 1994;118:16-23.

17. Weinreb RN, Zangwill L, Berry CC, et al. Detection of glaucoma with scanning laser polarimetry. Arch Ophthalmol. 1998:116: $1583-1589$

18. Hodapp E, Parrish R II, Anderson D. Clinical Decisions in Glaucoma. St. Louis: CV Mosby; 1993:11-63.

19. Johnson CA, Cioffi GA, Van Buskirk EM. Frequency doubling technology perimetry using a 24-2 stimulus presentation pattern. Optom Vis Sci. 1999;76:571-581.

20. Johnson CA, Gunvant P, Fingeret M, et al. A Primer for Frequency Doubling Technology (FDT) Perimetry Using the Humphrey Matrix. Zeiss: Welch-Allyn; 2004.

21. Delgado MF, Nguyen NT, Cox TA, et al. Automated perimetry: a report by the American Academy of Ophthalmology. Ophthalmology. 2002;109:2362-2374. 